University of Nebraska - Lincoln

DigitalCommons@University of Nebraska - Lincoln

Robert Katz Publications

Research Papers in Physics and Astronomy

February 1971

\title{
Simulation of Particle Tracks in Emulsion
}

Thomas E. Furtak

University of Nebraska-Lincoln, tfurtak@mines.edu

Robert Katz

University of Nebraska-Lincoln, rkatz2@unl.edu

Follow this and additional works at: https://digitalcommons.unl.edu/physicskatz

Part of the Physics Commons

Furtak, Thomas E. and Katz, Robert, "Simulation of Particle Tracks in Emulsion" (1971). Robert Katz Publications. 124.

https://digitalcommons.unl.edu/physicskatz/124

This Article is brought to you for free and open access by the Research Papers in Physics and Astronomy at DigitalCommons@University of Nebraska - Lincoln. It has been accepted for inclusion in Robert Katz Publications by an authorized administrator of DigitalCommons@University of Nebraska - Lincoln. 
Published in radiation effects 11 (1971), pp. 195-199. Copyright @ 1971 Gordon and Breach, Science Publishers Ltd. [Division of

Taylor \& Francis] Used by permission. http://www.tandf.co.uk/journals/titles/10420150.asp

Research supported by the U.S. Atomic Energy Commission and the National Science Foundation.

\title{
Simulation of Particle Tracks in Emulsion
}

\author{
T. E. Furtak and R. Katz \\ Behlen Laboratory of Physics, University of Nebraska, Lincoln, Nebraska 68508
}

\begin{abstract}
Micrographs of energetic particles in nuclear emulsion are simulated by computing the spatial distribution of developed grains with the $\delta$-ray theory of track structure, and representing the developed grains by points of light on an IBM 2250 visual display unit. The simulated tracks display many of the features of actual track structure and serve as a visual comparison between experiment and theory,
\end{abstract}

\section{Introduction}

The $\delta$-ray theory of charged particle track structure assumes that the response of small sensitive volumes lying on isodose contours near the path of an energetic ion is as if these volumes were part of a larger system uniformly irradiated with $\gamma$-rays to the same dose. Knowledge of the response function of a medium to $\gamma$-rays may be coupled to a knowledge of the spatial distribution of energy deposition to yield the spatial distribution of response.

In earlier work analyzing the effects of heavy ion bombardment on various detectors, this model has been successfully applied to the inactivation of dry enzymes and viruses, ${ }^{(1)}$ to survival of bacterial spores, yeast, and mammalian cells, ${ }^{(2)}$ to the formation of etchable tracks in dielectrics, ${ }^{(3)}$ to the response of $\mathrm{NaI}(1)$ scintillation counters, ${ }^{(4)}$ the silver activated phosphate glass dosimeter, the LiF thermoluminescent dosimeter, solid and liquid organic scintillators, the ferrous sulfate (Fricke) dosimeter, the creation of free radicals in solid biological substances, ${ }^{(5)}$ and to the formation of tracks in nuclear emulsion. ${ }^{(6,7)}$

The most detailed study of the spatial distribution of response can be achieved through use of nuclear emulsion; for in other detecting systems, the observed quantity is either the total response to the passage of a single particle, as in a scintillation counter, or the response of the system to the irradiation by a beam of particles. For this reason it is useful to reconstruct the tracks of ions in emulsion as a test of the theory.

In the present work the simulation of particle tracks in emulsion is achieved graphically through use of an IBM 2250 visual display unit. Developed grains are simulated by dots on the screen, whose positions are computed from the theory of the spatial distribution of response in emulsion. By altering the effective charge number of the ion $(z)$, its speed $(v=\beta c)$, and a parameter $\left(E_{0}\right)$ which describes the combined effect of sensitivity and processing, any track can be reconstructed. The simulated tracks correspond well to actual photomicrographs over a range of emulsion sensitivities for a variety of ions.

\section{Spatial Distribution of Response}

If $G$ is the number of grains per unit volume in the undeveloped emulsion, and $P$ is the probability that any one grain will become developed, then the number of developed grains per unit volume is the product $P G$. Determination of the spatial distribution of probability is the key to simulating track structure.

The emulsion is assumed to display a "one-hit" response, in which a single "action event" within the grain is responsible for sensitization. The probability of sensitization is described by the one-or-more hit Poisson formula

$$
P=1-\exp \left(-\bar{E} / E_{0}\right)
$$

where $\bar{E}$ is the mean dose deposited in the sensitive volume, and $E_{0}$ is the dose required for sensitization of 63 per cent of the available grains in a uniformly irradiated specimen of emulsion. The determination of the spatial distribution of probability follows from a determination of the spatial distribution of mean dose.

To evaluate the mean dose in a representative extended target, the point target dose as a function of 
the radial distance from the ion's path is determined following the method of Butts and Katz ${ }^{(1)}$ in which we assume normal ejection of $\delta$-rays, neglect binding energy, and take the electron to have constant energy loss, calculated for emulsion from an empirical algorithm of Kobetich and Katz. ${ }^{(8)}$ We take the electron energy $w$, to be related to the range $t$, by the equation $w=t / k$, where $k=3015 \mathrm{~cm} / \mathrm{erg}$, to sufficient accuracy in the dimensional regime of interest here. Using this simplified relation with the Butts-Katz theory, the point target dose at a radial distance $t$ from the ion's path is given by

$$
E=\frac{N z^{2} e^{4}}{m c^{2} \beta^{2}}\left(\frac{1}{t^{2}}-\frac{1}{t T}\right)
$$

$e$ and $m$ are the electron charge and mass, and $N$ is the electron number density, $1.04 \times 10^{24}$ electrons $/ \mathrm{cm}^{3}$ in emulsion, and $T$ is the range of the most energetic $\delta$ ray, calculated as in previous work from kinematic consideration. The effective charge number of an ion of atomic number $Z$ moving at speed $\beta c$ is given by Barkas $^{(9)}$ as $z=Z\left(1-\exp \left(-125 \beta Z^{-2 / 3}\right)\right)$.

The point target dose is averaged over a sensitive volume, approximated by a short (near spherical) cylinder of radius $a_{0}$, whose axis is parallel to and at a radial distance $t$ from the path of an ion. The best "fit" of the present simulation to actual track photographs is obtained with $a_{0}$ equal to 0.17 microns, taken to represent the effective radius of an unsensitized grain.

The mean dose determined in this manner and the value of $E_{0}$ assigned by trial for best "fit" of the simulated to the observed tracks are used in Equation (1) to determine the spatial distribution of response.

\section{A Model for Track Simulation}

The depth of a simulated emulsion ( $d$ in Figure 1) must approximate the depth, before processing, of that part of an actual emulsion in sharp focus in a microphotograph. Here we have taken $d$ to be $0.25 \mu$, as an estimate of the combined effects of depth of focus, shrinkage factor, and perception. For calculation, we consider the available emulsion to consist of long box-shaped sub-volumes of length $L$. The center subvolume $(A)$ is chosen so that the ion passes along its axis. Sub-volumes $B$ and $B^{\prime}, C$ and $C^{\prime}, \ldots$ are symmetrically chosen on opposite sides of the path. Each box-shaped sub-volume is further divided into equal microboxes, as shown in the first quadrant of sub-volume $A$. The number of grains available for sensitization per micro-box is the product of the grain density $(G)$, the length of the track segment $(L)$, and the cross

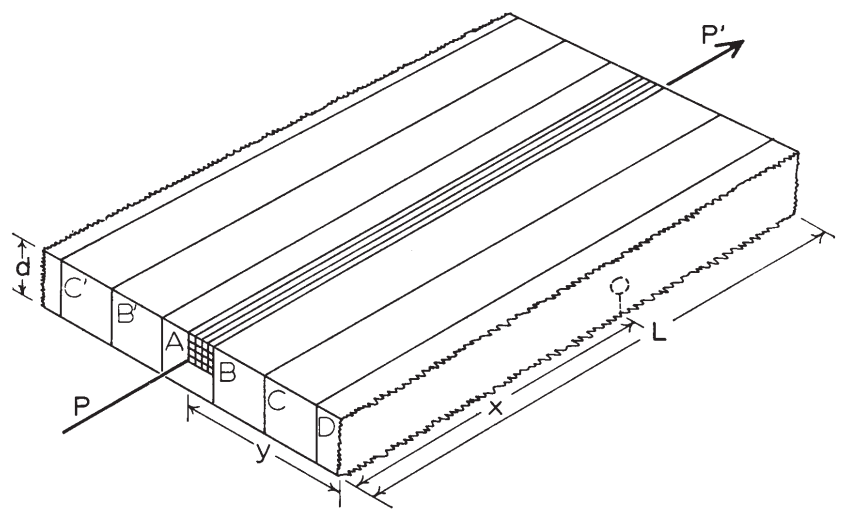

Figure 1. A schematic representation of the simulated emulsion. The depth and length of the track segment are the distances $d$ and $L$. The ion follows the path $P P^{\prime}$. The position of a developed grain in sub-volume $D$ defines a set of $x-y$ coordinates for that grain as shown.

section of the micro-box. When simulating tracks in Ilford $G$ emulsion a grain density of $4.9 \times 10^{13}$ grains / $\mathrm{cm}^{3}$ is assumed, while for Ilford $K$ emulsion a grain density of $1.0 \times 10^{14}$ grains $/ \mathrm{cm}^{3}$ is used. Values of undeveloped grain density and emulsion sensitivity $\left(E_{0}\right)$ are the only parameters used here for describing the characteristics of different emulsions. No attempt is made to change the effective undeveloped grain size in this preliminary investigation when simulating tracks in emulsion having a different grain size from that of Ilford $G$ emulsion.

Equation (1) is used to determine the probability of sensitizing grains for each micro-box within a subvolume. The probability in the separate micro-boxes making up a sub-volume is summed, multiplied by the number of available undeveloped grains in the sub-volume, and rounded to an integral value which represents the number of developed grains located within the sub-volume. These grains are taken to lie along the axis of the sub-volume, thus defining their $y$-coordinate (see Figure 1), and have randomized positions chosen by a computer algorithm, thus defining their $x$-coordinates. This procedure gives the spatial distribution of grains about the path of the ion in Cartesian coordinates.

The matrix of coordinate pairs representing the distribution of developed grains is transformed to a matrix of points of light by an IBM 2250 visual display unit coupled (in a direct interaction mode) to an IBM 360 model 65 computer system. The scale is adjusted to compensate for the diameter of a developed grain, taken to be $0.7 \mu$ for both $G$ and $K$ emulsion. Photomicrographs of a track segment in emulsion can be simulated by varying the parameters $E_{0}, Z$, and $\beta$. 


\section{Results}

Our previous investigations of particle tracks in emulsion ${ }^{(6)}$ examined separately either grain counts or track widths, except for a recent analysis of a breakup event, ${ }^{(7)}$ where particle charge and velocity were positively identified, which demonstrated the consistency of the grain count work with the track width work. Only in the region of a breakup can we say with certainty that the emulsion sensitivity is the same for all participating tracks.

Reconstruction of that breakup event is used by this work to test the validity of the simulation. The experimental breakup event on the left-hand side of Figure 2 has been previously identified as an oxygen nucleus fragmenting into a carbon nucleus, two protons, and neutrons. Range measurements on the proton tracks show the particles to be travelling at a

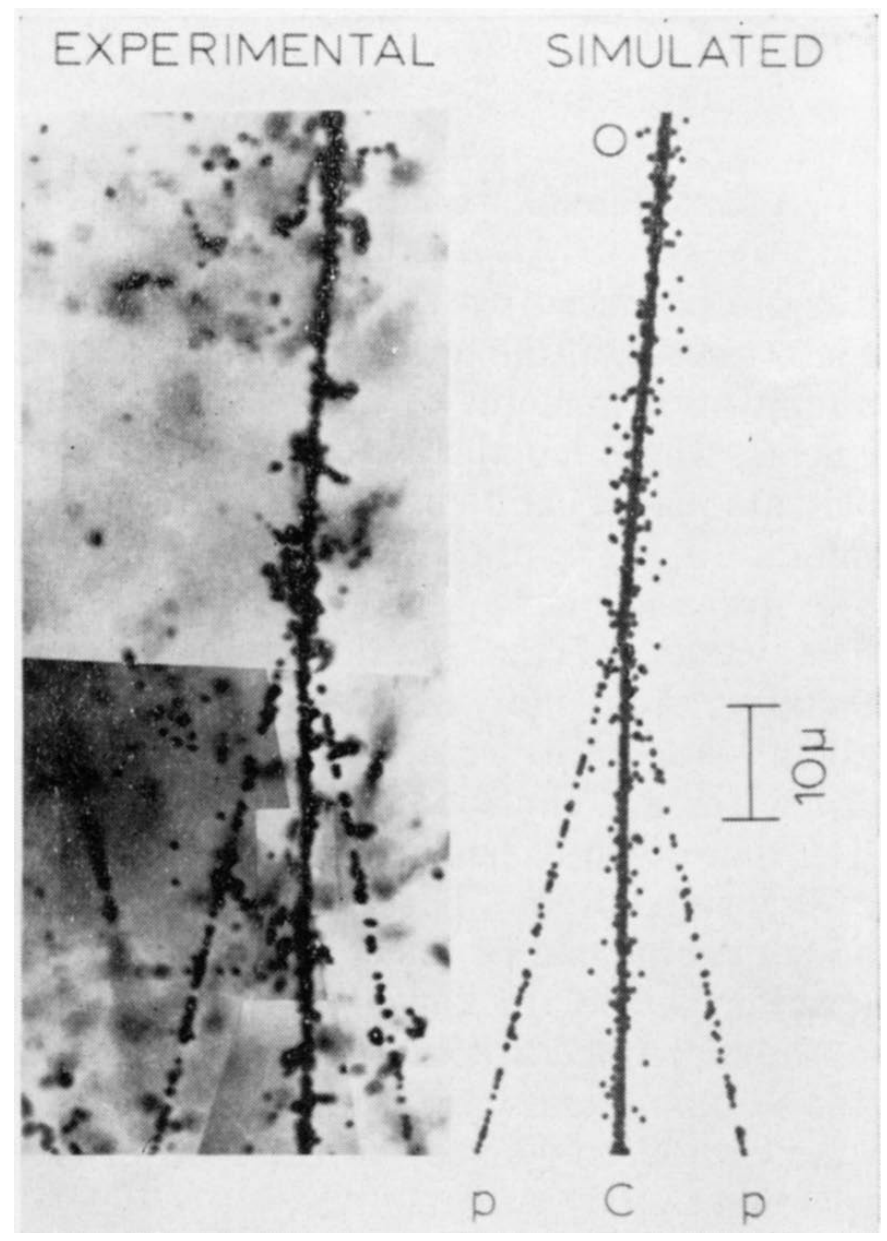

Figure 2. Montage of tracks simulating the breakup of an oxygen nucleus into a carbon nucleus, two protons, and neutrons. The analysis of a breakup is the best test of the simulation in both the grain count and track width regimes, since the emulsion sensitivity must be constant. speed of $0.32 c$ at breakup. Inserting the appropriate values of $\beta$ and $Z$ and using an emulsion sensitivity of $E_{0}=64,000 \mathrm{ergs} / \mathrm{cm}^{3}$, the four simulated tracks on the right hand side of Figure 2 were reassembled to match the actual event from tracks individually displayed on the 2250 screen and photographed. Grain counts, converted by statistical methods from experimental blob counts of the grainy fragments, ${ }^{(7)}$ show approximately 125 grains/100 $\mu$ at breakup. The grain count from the computer simulation for the two protons is 129 grains $/ 100 \mu$. In the analysis of the experimental primary and heavy fragment, the area of individual linear sections, traced with pen and ink and measured with a planimeter, was divided by the length of the section to find a value for mean track width. The same method used on the simulated tracks confirms the fit of the simulation in the track width regime.

In Figure 3, relativistic cosmic ray particles (identified as $Z=1,7$, and 20) in Ilford G.5 emulsion processed to similar grain count at minimum ionization, from the Naval Research Laboratory, are displayed alongside their simulations. The value of $E_{0}=44,000$ ergs $/ \mathrm{cm}^{3}$ is "fitted" to the proton grain count assuming a velocity of $0.95 \mathrm{c}$. The transition from the

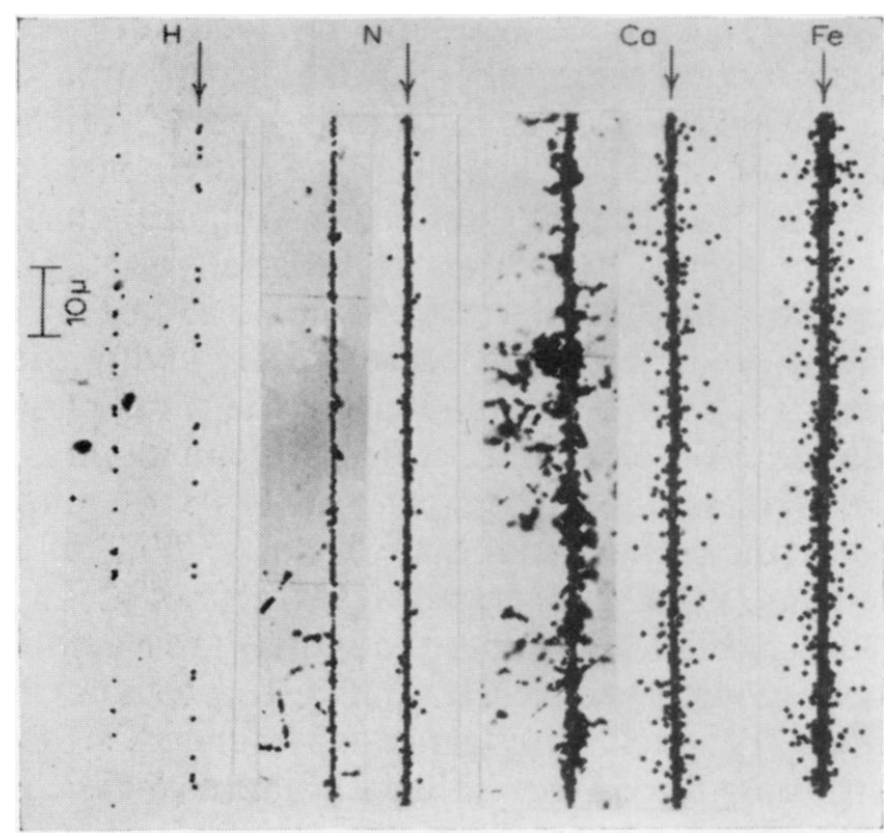

Figure 3. Relativistic hydrogen, nitrogen, and calcium ions in Ilford G.5 emulsion (courtesy M. M. Shapiro). Simulated tracks are designated by arrows. Note the transition from the grain count regime to the track width regime with no change in the method of simulation. A simulated iron track yields a better description of the "globbed" structure of the experimental Ca track than does the simulated Ca track. 


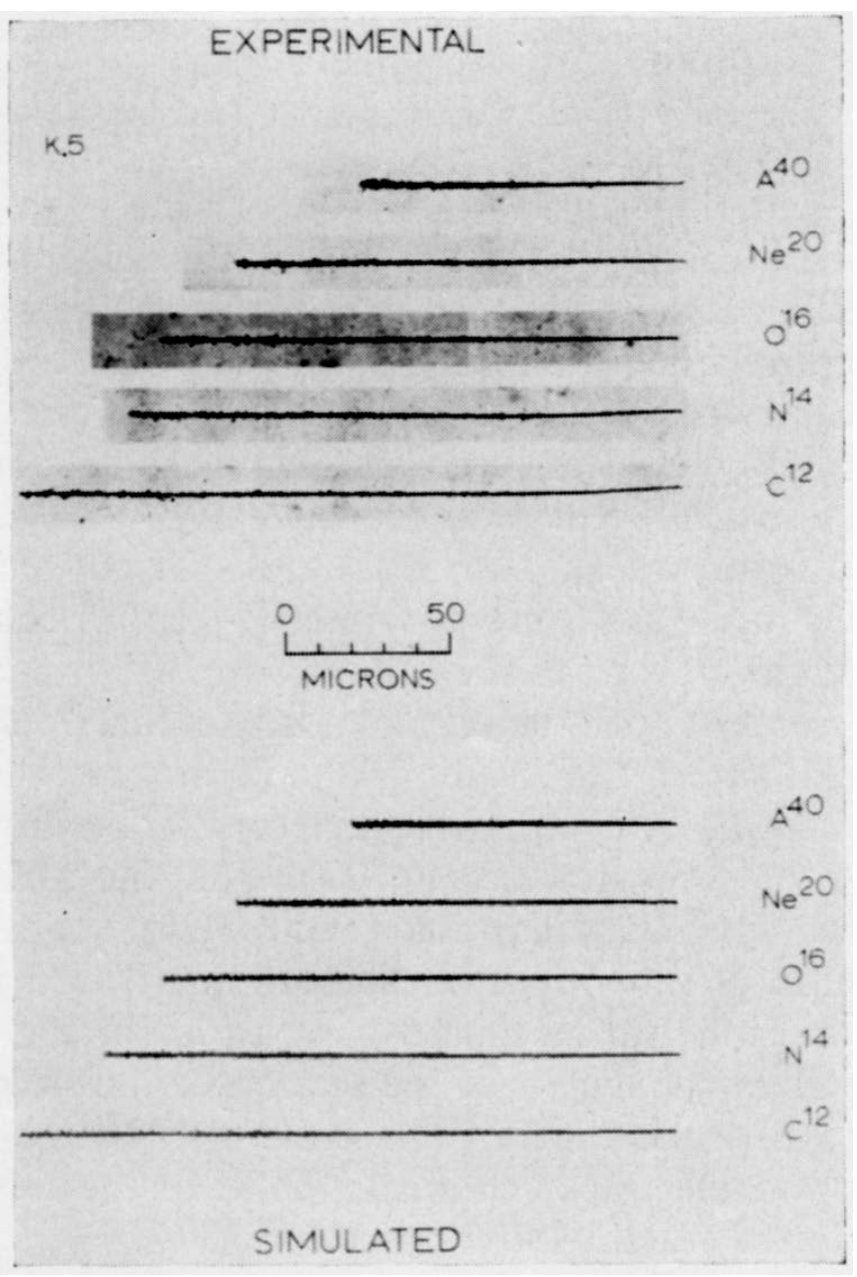

Figure 4. Stopping ${ }^{40} \mathrm{~A},{ }^{20} \mathrm{Ne},{ }^{16} \mathrm{O},{ }^{14} \mathrm{~N}$, and ${ }^{12} \mathrm{C}$ ions of initial energy $10.5 \mathrm{MeV} / \mathrm{amu}$, in Ilford K.5 emulsion. The "bumpy" appearance, thin-down, grainy and "globbed" structure of the experimental photographs are all demonstrated in the simulation. Courtesy E. V. Benton.

grain count regime to the track width regime is illustrated well. The only parameter which is changed is the atomic number, $\mathrm{Z}$. The absence of "globbed" structure on the calcium simulation raises a question about the identification of that experimental track or of its simulation, if the emulsion sensitivity and particle speed are taken to be uniform in the three photographs. Note how more closely the simulated iron track resembles the experimental photograph. This result is obtained if the value of $\left(z^{2} / \beta^{2} E_{0}\right)$ of the simulated track is increased by a factor of $(26 / 20)^{2}$.

The simulation assumes that grains are randomly placed along the track. It is interesting to note how closely the track appearance is simulated without attempting to connect isolated grains as $\delta$-rays.

Photographs of tracks of ${ }^{40} \mathrm{~A},{ }^{20} \mathrm{Ne},{ }^{16} \mathrm{O},{ }^{14} \mathrm{~N}$, and ${ }^{12} \mathrm{C}$ ions, of initial energy $10.5 \mathrm{MeV} / \mathrm{amu}$, in Ilford K.5, K.0, and K-2 emulsion, from Benton and Toch-

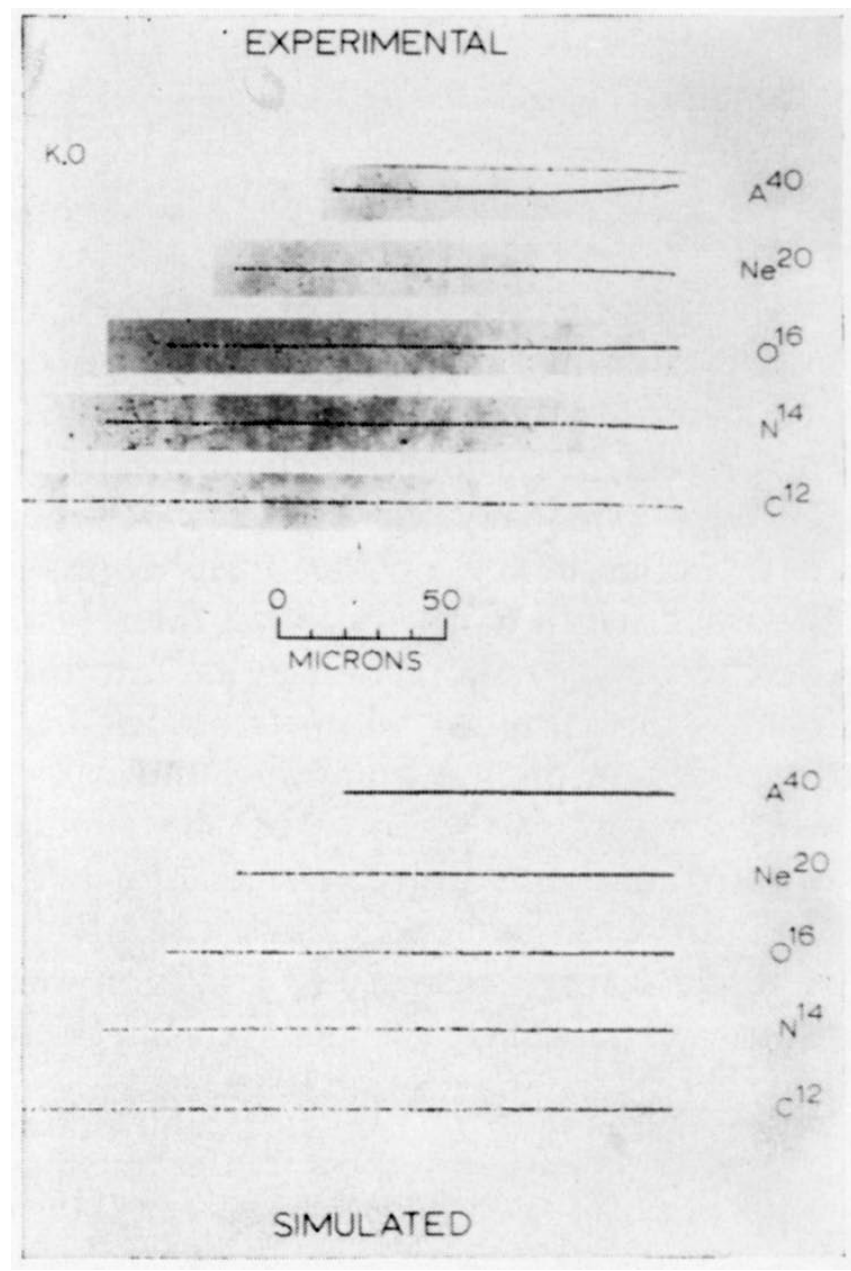

Figure 5. Ilford K.0 emulsion. See Figure 4.

ilin,, ${ }^{(10)}$ are shown in Figures 4-6. The simulated tracks are produced by decreasing $\beta$ from 0.15 , on the left, to 0.02 , at the termination of the tracks on the right, in increments of $\Delta \beta=0.01$. Constant $\beta$ segments, whose lengths are computed from range tables, are placed end to end by the computer. The resultant display on the IBM 2250 is representative of the stopping tracks.

The values of the parameter $E_{0}$ chosen are 200,000 $\mathrm{ergs} / \mathrm{cm}^{3}$ for $K .5$ emulsion, $24,000,000 \mathrm{ergs} / \mathrm{cm}^{3}$ for $K .0$ emulsion, and $320,000,000 \mathrm{ergs} / \mathrm{cm}^{3}$ for $\mathrm{K}-2$ emulsion.

The thin-down of heavy ions is demonstrated in the K.5 simulation. The bumpy appearance at the high energy end of the tracks is also apparent. The difference in track width between the actual and the simulated tracks, particularly noticeable at the stopping tip, is due primarily to the spot size on the cathode ray screen of the IBM 2250 visual display unit. 


\section{Discussion}

Preliminary tests of the track simulation procedure indicate that a proper direction for further applications has been defined.

Some doubt still exists as to the precision with which the spatial distribution of energy (and therefore, the value of $E_{0}$ ) is known. The procedure followed in this work was chosen for economy and convenience in computation.

The simulation makes possible a direct visual comparison of theoretical with experimental results. Not only does it serve as another test of the $\delta$-ray theory of track structure, but it also promises to lead to the use of tracks in emulsion as an experimental means of determining the spatial distribution of ionization energy about the path of an energetic ion, reversing the original roles of theory and experiment in track studies.

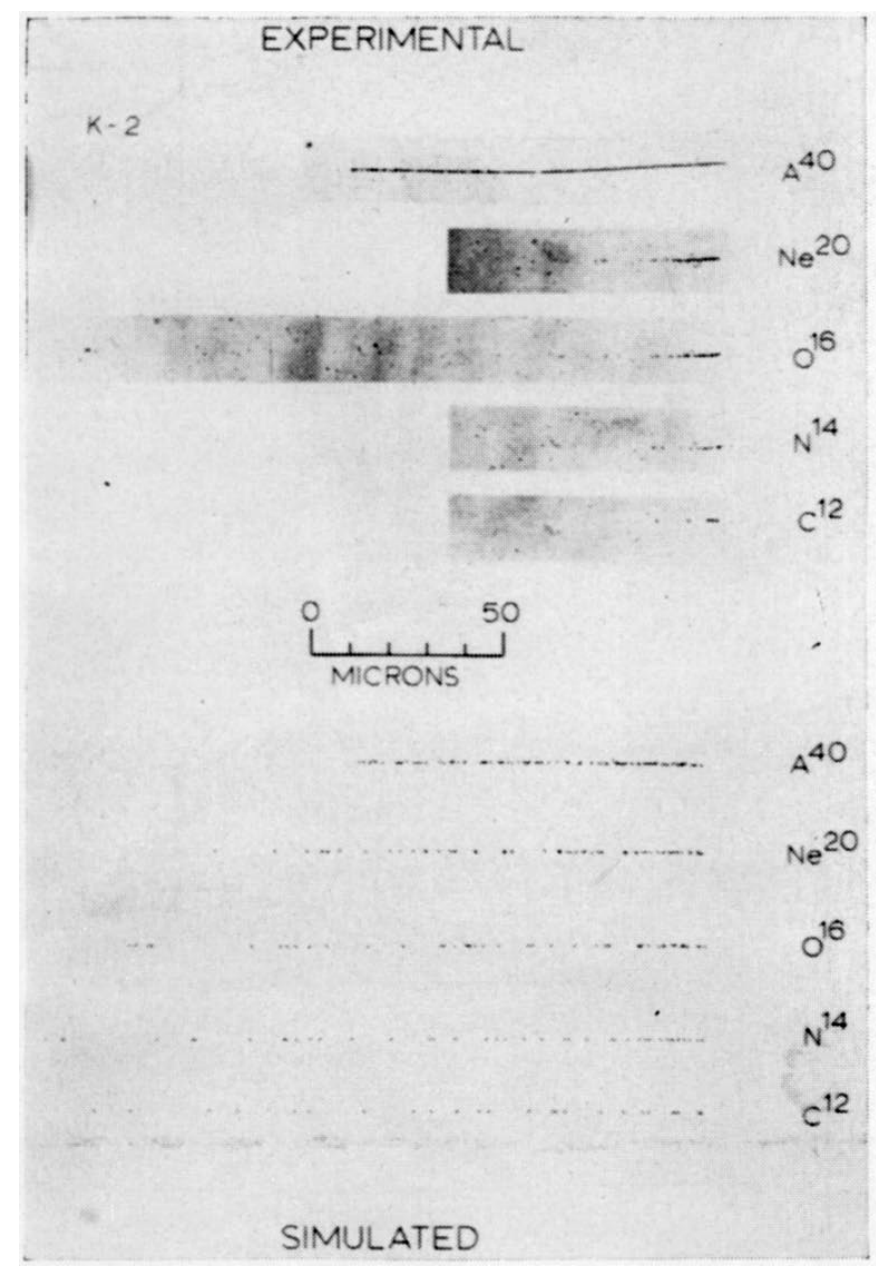

Figure 6. Ilford K-2 emulsion. See Figure 4.

\section{References}

1. J. J. Butts and R. Katz, Rad. Res., 30, 855 (1967)

2. R. Katz, B. Ackerson, M. Homayoonfar, and S. C. Sharma, Rad. Res., 47, 402 (1971).

3. R. Katz and E. J. Kobetich, Phys. Rev., 170, 401 (1968).

4. R. Katz and E. J. Kobetich, Phys. Rev., 170, 397 (1968).

5. R. Katz, S. C. Sharma, and M. Homayoonfar, 'Detection of Energetic Heavy Ions' to be published.

6. R. Katz and E. J. Kobetich, Phys. Rev., 186, 344 (1969).

7. B. Ackerson, C. M. Sorensen, and R. Katz, Nucl. Inst. Methods, 92, 81 (1971).

8. E. J. Kobetich and R. Katz, Nucl. Inst. Methods, 71, 226 (1969).

9. W. H. Barkas, Nuclear Research Emulsions, Volume 1, Academic Press, Inc., New York (1963).

10. E. V. Benton and E. Tochilin, Health Phys., 12, 49 (1966). 